\title{
Non-axisymmetric spherical interface dynamos
}

\author{
K. H. Chan ${ }^{1}$, X. Liao ${ }^{2}$, K. Zhang ${ }^{1}$, and C. A. Jones ${ }^{1}$ \\ 1 Department of Mathematical Sciences, Laver Building, University of Exeter, EX4 4QE, UK \\ e-mail: kzhang@ex.ac.uk \\ 2 Shanghai Astronomical Observatory, Chinese Academy of Sciences, Shanghai 200030, PR China
}

Received 4 June 2004 / Accepted 12 July 2004

\begin{abstract}
A fully three-dimensional, nonlinear, time-dependent spherical interface dynamo with a solar-like internal differential rotation and a single-cell meridional circulation is investigated. There are four regions in our interface dynamo model: a uniformly rotating core, a thin differentially rotating tachocline, a turbulent convection zone and a nearly insulating exterior. The different regions are coupled by the electric and magnetic matching conditions at the interfaces. For the first time, nonaxisymmetric spherical nonlinear interface dynamos are found. When the magnetic Reynolds number based on the differential rotation is small or when the effect of the meridional circulation is substantial, we show that the spherical interface dynamos are non-axisymmetric even though the $\alpha$-distribution, differential rotation and meridional circulation are all axisymmetric. Implication of the results for the solar dynamo is discussed.
\end{abstract}

Key words. magnetohydrodynamics - Sun: interior - Sun: magnetic field

\section{Introduction}

A highly differentially rotating transition zone between the convection and radiative regions of the Sun, the solar tachocline, plays an essential role in generating large-scale solar magnetic activities such as the $11 \mathrm{yr}$ sunspot cycle (e.g., Spiegel \& Zahn 1992; Parker 1993; Tobias et al. 1995). It is the tachocline that offers an ideal location for both the generation and storage of the Sun's strong azimuthal magnetic fields, leading to the first cartesian interface dynamo model proposed by Parker (1993). A key idea in an interface dynamo is that the production of a weak poloidal magnetic field and a strong azimuthal magnetic field occurs in separate fluid regions with discontinuous magnetic diffusivities across their interface.

There exist a number of spherical interface dynamo models (e.g. Charbonneau \& MacGregor 1997; Markiel \& Thomas 1999; Dikpati \& Charbonneau 1999; Covas et al. 2001). Two important assumptions have been employed in nearly all the existing spherical interface dynamos. They assumed that the generated magnetic field $\boldsymbol{B}$ is axisymmetric, usually in the form of

$\boldsymbol{B}=B_{\phi}(r, \theta, t) \hat{\boldsymbol{\phi}}+\nabla \times[A(r, \theta, t) \hat{\boldsymbol{\phi}}]$.

They also assumed the interface dynamos are of $\alpha \Omega$-type, i.e., the alpha term in the $B_{\phi}$ equation is neglected. The numerical analysis is simplified as a result of the above two assumptions. However, an axisymmetric alpha-profile and an axisymmetric large-scale flow (the differential rotation and meridional circulation) do not necessarily lead to an axisymmetric spherical interface dynamo.
The recent solar observations suggest that the solar dynamo is strongly non-axisymmetric, characterized by magnetic activities in persistent different longitudes and the total number of active regions per rotation per hemisphere varied between zero and seven (e.g., De Toma et al. 2000). Non-axisymmetry of the solar magnetic fields may be attributable to the joint instability of latitudinal differential rotation and concentrated magnetic field in the tachocline, which can produce non-axisymmetric magnetic fields with longitudinal wavenumners up to $m=5$ (e.g., Gilman \& Fox 1997; Gilman \& Dikpati 2000; Zhang et al. 2003). De Toma et al. (2000) argued that the number of persistent bands of magnetically active nests in the observed solar magnetic fields corresponds to the longitudinal wavenumber $m$ in the magnetic instability problem.

The primary objective of this letter is to report a new interface dynamo phenomenon discovered in the numerical simulation of a fully three-dimensional finite-element spherical interface dynamo. We found that the spherical interface dynamos with an axisymmetric alpha-effect and an axisymmetric flow (the differential rotation and meridional circulation) are nonaxisymmetric when the magnetic Reynolds number based on the differential rotation is small or when the effect of the meridional circulation is substantial.

\section{Interface dynamo model}

Our spherical interface dynamo model consists of four different zones. The inner radiative sphere, $0<r<r_{i}$, has a very small magnetic diffusivity and rotates uniformly with the angular velocity $\boldsymbol{\Omega}_{\mathrm{i}}$. We take the reference of frame that rotates with $\boldsymbol{\Omega}_{\mathrm{i}}$. 
On the top of the radiative core, a strong differential rotation with a large radial shear is confined in the tachocline $r_{\mathrm{i}}<r<r_{\mathrm{t}}$ with magnetic diffusivity $\lambda_{\mathrm{t}}$. Note that magnetic fields cannot be generated by the differential rotation even in the presence of radially discontinuous magnetic diffusivities (e.g., Zhang \& Liao 2003). However, the weak poloidal magnetic field diffuses down into the tachocline where it is strongly sheared, resulting in a strong magnetic field $\boldsymbol{B}_{\mathrm{t}}(\theta, r, \phi, t)$. This process is described by the following equations

$\frac{\partial \boldsymbol{B}_{\mathrm{t}}}{\partial t}=\nabla \times\left(\boldsymbol{U}_{\Omega}(\theta, r) \times \boldsymbol{B}_{\mathrm{t}}\right)+\lambda_{\mathrm{t}} \nabla^{2} \boldsymbol{B}_{\mathrm{t}}$,

$\nabla \cdot \boldsymbol{B}_{\mathrm{t}}=0$

where $\boldsymbol{U}_{\Omega}(\theta, r)$ is the solar-like differential rotation (Schou et al. 1998). We assume a fully turbulent convection in the region $r_{\mathrm{t}}<r<r_{\mathrm{o}}$. In this zone, a weak magnetic field $\boldsymbol{B}_{\mathrm{o}}(\theta, r, \phi, t)$ is generated by an $\alpha^{2} \omega$ dynamo (both the $\alpha$ and $\omega$ are axisymmetric). We assume an eddy magnetic diffusivity $\lambda_{\mathrm{o}}$ in the convection zone, taking the ratio $\lambda_{\mathrm{t}} / \lambda_{\mathrm{o}}=10^{-2}$ throughout the paper. The $\alpha^{2} \omega$ dynamo in the convection zone is described by

$$
\begin{aligned}
\frac{\partial \boldsymbol{B}_{\mathrm{o}}}{\partial t}= & \alpha_{\mathrm{o}} \nabla \times \alpha\left(r, \theta,\left|\boldsymbol{B}_{\mathrm{o}}\right|^{2}\right) \boldsymbol{B}_{\mathrm{o}} \\
& +\nabla \times\left(\boldsymbol{U}_{\Omega}(\theta)+\boldsymbol{U}_{\mathrm{p}}(\theta, r)\right) \times \boldsymbol{B}_{\mathrm{o}}+\lambda_{\mathrm{o}} \nabla^{2} \boldsymbol{B}_{\mathrm{o}},
\end{aligned}
$$

$\nabla \cdot \boldsymbol{B}_{\mathrm{o}}=0$,

where $\alpha_{\mathrm{o}}$ is a parameter, $\boldsymbol{U}_{\mathrm{p}}(\theta, r)$ represents a single-cell meridional circulation having a poleward surface flow (Dikpati \& Charbonneau 1999; Küker et al. 2001). The nonlinear function $\alpha\left(r, \theta,\left|\boldsymbol{B}_{\mathrm{o}}\right|^{2}\right)$ is associated with the local $\alpha$-quenching (Brandenburg 1994) and is of the form

$\alpha\left(r, \theta,\left|\boldsymbol{B}_{\mathrm{o}}\right|^{2}\right)=\sin ^{2} \theta \cos \theta \sin \left[\pi \frac{\left(r-r_{\mathrm{t}}\right)}{\left(r_{\mathrm{o}}-r_{\mathrm{t}}\right)}\right] \frac{1}{\left(1+\left|\boldsymbol{B}_{\mathrm{o}}\right|^{2}\right)}$

in $r_{\mathrm{t}}<r \leq r_{\mathrm{o}}$, which satisfies $\alpha\left(r_{\mathrm{t}}, \theta,\left|\boldsymbol{B}_{\mathrm{o}}\right|^{2}\right)=\alpha\left(r_{\mathrm{o}}, \theta,\left|\boldsymbol{B}_{\mathrm{o}}\right|^{2}\right)=$ 0 . The outer exterior to the convection zone is assumed to be nearly electrically insulating. The magnetic and electric matching conditions at the interfaces couple the various zones together. The detailed numerical method for our spherical interface dynamo was discussed by Zhang et al. (2003).

\section{Results of the interface dynamo simulation}

Three non-dimensional quantities characterize our spherical interface dynamo model: the magnetic alpha Reynolds number $R_{\alpha}$ and the magnetic Reynolds number $R_{\mathrm{m}}$ based on the differential rotation, the magnetic Reynolds number $R_{\mathrm{p}}$ based on the meridional circulation, which are defined by

$R_{\alpha}=\frac{\left(r_{\mathrm{o}}-r_{\mathrm{i}}\right) \alpha_{\mathrm{o}}}{\lambda_{\mathrm{o}}}, R_{\mathrm{m}}=\frac{\left(r_{\mathrm{o}}-r_{\mathrm{i}}\right) U_{\Omega}}{\lambda_{\mathrm{o}}}, R_{\mathrm{p}}=\frac{\left(r_{\mathrm{o}}-r_{\mathrm{i}}\right) U_{\mathrm{p}}}{\lambda_{\mathrm{o}}}$,

where $U_{\Omega}$ is the speed of the differential rotation at the equator in the rotating frame with $\boldsymbol{\Omega}_{\mathrm{i}}$ and $U_{\mathrm{p}}$ represents the typical speed of the meridional circulation. Since $\lambda_{\mathrm{o}}$ and $U_{\mathrm{p}}$ are not well known, we shall treat $R_{\alpha}, R_{\mathrm{m}}$ and $R_{\mathrm{p}}$ as varying parameters in our simulations.
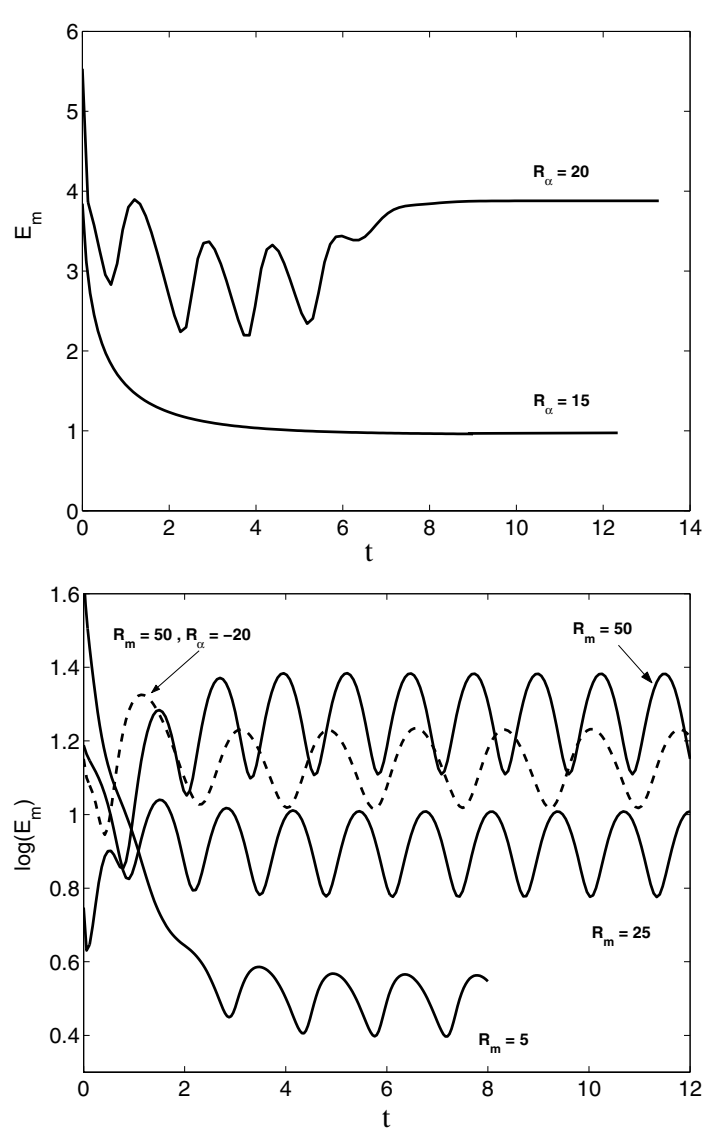

Fig. 1. Magnetic energy $E_{\mathrm{m}}$ of the generated magnetic field is shown as a function of time when $R_{\mathrm{p}}=0$ : the upper panel shows dynamos with $R_{\mathrm{m}}=0$ and the lower panel shows the effects of the differential rotation for a range of $R_{\mathrm{m}}$ at $R_{\alpha}=20$ (solid lines). The dynamo solution for a negative $R_{\alpha}=-20$ is shown by the dashed line.

When $R_{\mathrm{p}}=0$ and $R_{\mathrm{m}}=0$, i.e., the generation of the magnetic field without the effects of both the differential rotation and the meridional circulation, the resulting dynamo corresponds to a conventional $\alpha^{2}$ dynamo driven by an axisymmetric $\alpha$ in the convection zone. It should be noted that our simulation does not impose any azimuthal or equatorial symmetries. By performing simulations at various values of $R_{\alpha}$, it is found that the dynamo action first occurs at about $R_{\alpha}=12$. In Fig. 1 (upper panel), we show the magnetic energy $E_{\mathrm{m}}$ of the generated magnetic field as a function of time for two different $R_{\alpha}$. The structure of the generated magnetic field for the stationary dynamo at $R_{\alpha}=20$ is depicted in Fig. 2. An important new feature of the spherical dynamo is that the conventional nonlinear dynamo is always non-axisymmetric with the azimuthal wavenumber $m=4$, regardless of the spatial properties of the initial condition. The non-axisymmetry of the spherical dynamo can be explained by the effect of the nearly perfectly conducting core which blocks the effective communication of the generated magnetic field between the different regions of longitudes, leading to the preference of a non-axisymmetric dynamo mode.

When $R_{\mathrm{m}}$ is non-zero but $R_{\mathrm{p}}=0$, i.e., the generation of the magnetic field is affected by the differential rotation, the resulting dynamo corresponds to an $\alpha^{2} \omega$ dynamo driven by an 


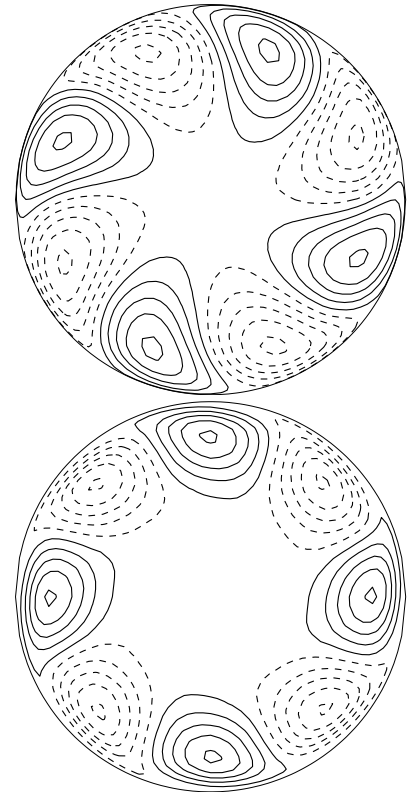

Fig. 2. Contours of $B_{\phi}$ on the spherical surface at $r=r_{\mathrm{t}}$ (upper panel) and $B_{r}$ on the spherical surface at $r=\left(r_{\mathrm{t}}+r_{\mathrm{o}}\right) / 2$ (lower panel) for $R_{\alpha}=$ 20 and $R_{\mathrm{m}}=R_{\mathrm{p}}=0$, viewed from the north pole. The contour levels are from $\left(B_{\phi}\right)_{\min }=-0.6$ to $\left(B_{\phi}\right)_{\max }=+0.6$ and from $\left(B_{r}\right)_{\min }=-0.61$ to $\left(B_{r}\right)_{\max }=+0.61$. Solid (dashed) contours indicate $B_{\phi}>0$ or $B_{r}>0$ $\left(B_{\phi}<0\right.$ or $\left.B_{r}<0\right)$.

axisymmetric $\alpha$ and influenced by the differential rotation. We have performed a number of simulations for different values of $R_{\mathrm{m}}$, which are shown in Fig. 1 (lower panel). It is found that the spherical dynamos are still non-axisymmetric with the azimuthal wavenumber $m=4$ when $R_{\mathrm{m}}$ is insufficiently large, $R_{\mathrm{m}} \leq 5$. Figure 3 shows the structure of the generated magnetic field for $R_{\alpha}=20$ with $R_{\mathrm{m}}=1$ on a spherical surface. When $R_{\mathrm{m}} \geq O(10)$, however, the effective communication of the generated magnetic field between the different regions of longitudes is re-established by the differential rotation, leading to a periodic axisymmetric interface dynamo. In this case, strong azimuthal field is generated in the tachocline and the magnetic field propagates radially toward the surface of the convection zone, regardless of whether $R_{\alpha}>0$ or $R_{\alpha}<0$. The typical structure of the radially propagating magnetic field for a positive $R_{\alpha}=20$ is depicted in Fig. 4 in a meridional plane.

When both $R_{\mathrm{m}}$ and $R_{\mathrm{p}}$ are non-zero, the resulting dynamo corresponds to an $\alpha^{2} \omega$ dynamo driven by an axisymmetric $\alpha$ and affected by both the differential rotation and meridional circulation. An important effect of the large-scale meridional circulation is to reduce the effective communication of the generated magnetic field between the different regions of longitudes. The interface dynamos become non-axisymmetic, in the form of azimuthally propagating dynamo waves, when $R_{\mathrm{p}}$ is sufficiently large. At any fixed meridional plane, as shown in the butterfly diagram in Fig. 5, the dynamo wave appears to propagate toward the equator. However, this only reflects an azimuthally propagating non-axisymmetric dynamo wave with the wavenumber $m=1$. The azimuthal structure of the generated toroidal field for $R_{\mathrm{m}}=50$ and $R_{\mathrm{p}}=25$ as a function of time is displayed in Fig. 6 at the interface between the

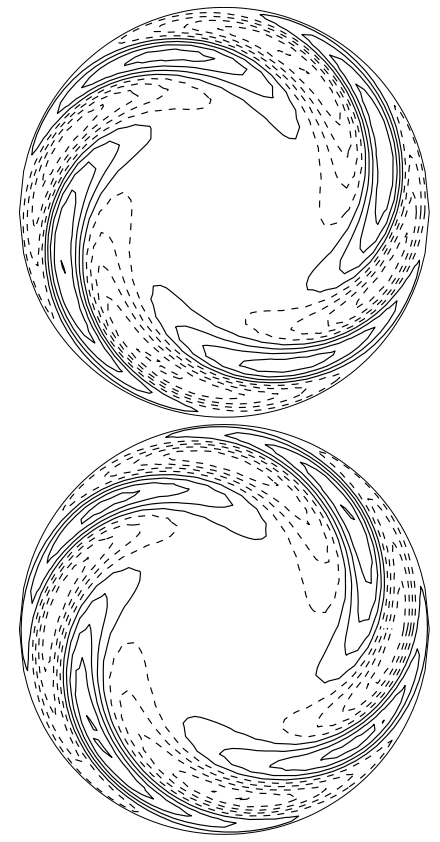

Fig. 3. Contours of $B_{\phi}$ on the spherical surface at $r=r_{\mathrm{t}}$ (upper panel) and $B_{r}$ on the spherical surface at $r=\left(r_{\mathrm{t}}+r_{\mathrm{o}}\right) / 2$ (lower panel) for $R_{\alpha}=$ $20, R_{\mathrm{m}}=1$ and $R_{\mathrm{p}}=0$, viewed from the north pole. The contour levels are from $\left(B_{\phi}\right)_{\min }=-0.51$ to $\left(B_{\phi}\right)_{\max }=+0.51$ and from $\left(B_{r}\right)_{\min }=$ -0.43 to $\left(B_{r}\right)_{\max }=+0.43$.

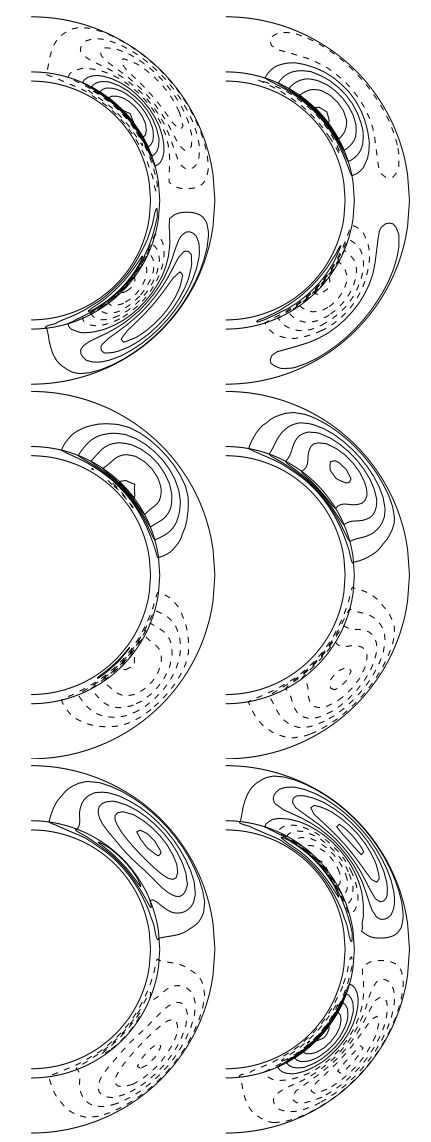

Fig. 4. Contours of $B_{\phi}$ in a meridional plane, plotted at six different instants in the half period (from left to right and top to bottom) for $R_{\alpha}=$ $20, R_{\mathrm{m}}=50, R_{\mathrm{p}}=0$. 


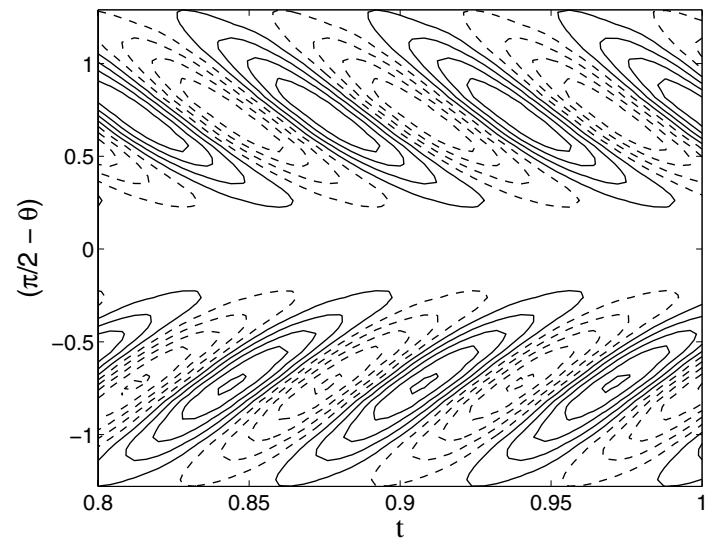

Fig. 5. A butterfly diagram for the dynamo solution at a fixed longitude with $R_{\alpha}=20, R_{\mathrm{p}}=25, R_{\mathrm{m}}=50$ where the azimuthal magnetic field $B_{\phi}$ evaluated at the interface between the tachocline and convection zone.

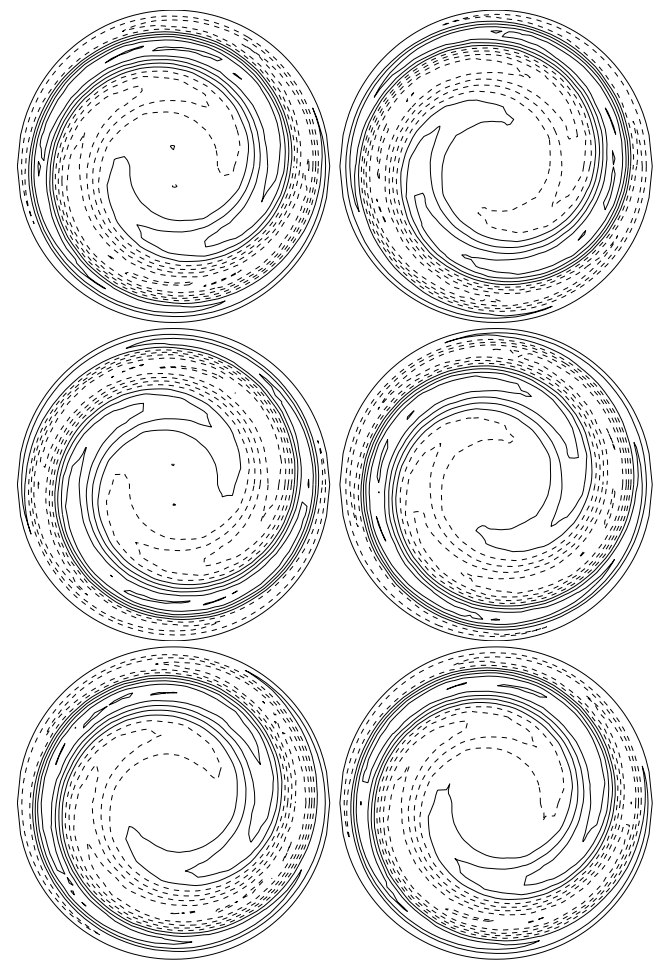

Fig. 6. Contours of $B_{\phi}$ is shown at the interface between the tachocline and convection zone for six different instants (from left to right and top to bottom) for $R_{\alpha}=20, R_{\mathrm{p}}=25, R_{\mathrm{m}}=50$, viewed from the north pole.

tachocline and convection zone where the toroidal field is the strongest. In a real system, a strong toroidal field stored in the tachocline in a particular longitudinal region would be susceptible to magnetic buoyancy leading to an eruption or other magnetic activities in the longitudinal region near the outer surface of the convection zone (Ferriz-Mas et al. 1994).

\section{Summary and remarks}

This paper presents the first non-axisymmetric spherical interface dynamos found in a fully three-dimensional, nonlinear dynamo model using a finite element method with threedimensional tedrahedralization in the spherical system. An important finding is that non-axisymmetric dynamo modes can be preferred in a spherical interface dynamo even though both the alpha-profile and large-scale flow (the differential rotation and meridional circulation) are axisymmetric. This provides a possible explanation of non-axisymmetry of the observed solar magnetic fields (De Toma et al. 2000).

Our interface dynamo model follows the classical picture of Parker (1955) in that the $\alpha$-effect is assumed to be operated throughout the convection zone. Alternatively, an interface dynamo may restrict the $\alpha$-effect near the surface of the Sun (Babcock 1961) or near the base of the convection zone resulting from the hydromagnetic or hydrodynamic instabilities taking place in the tachocline (e.g., Dikpati \& Gilman 2001). An investigation into the effect of different locations of the alphaprofile on a nonlinear spherical three-dimensional interface dynamo is currently under way.

Acknowledgements. This work is supported by UK PPARC and NERC grants. X.L. is supported by the Royal Society and NSFC grants. K.Z. would like to thank Dr. S. Tobias and Professor M. Proctor for many helpful discussions about the solar dynamo problem.

\section{References}

Babcock H. W. 1961, ApJ, 133,572

Brandenburg, A. 1994, in Lectures on Solar and Planetary Dynamos, ed. M. R. E. Proctor, \& A. D. Gilbert (Cambridge: CUP)

De Toma, G., White, O. R., \& Harvey, K. L. 2000, ApJ, 529, 1101

Charbonneau, P., \& MacGregor, K. B. 1997, ApJ, 486, 502

Covas, E., Tavakol, R., \& Moss, D. 2001, A\&A, 371, 718

Dikpati, M., \& Charbonneau, P. 1999, ApJ, 518, 508

Dikpati, M., \& Gilman, P. 2001, ApJ, 559, 428

Ferriz-Mas, A., Schmitt, D., \& Schüssler, M. 1994, A\&A, 289, 949

Gilman, P. A., \& Fox, P. A. 1997, ApJ, 484, 439

Gilman, P. A., \& Dikpati, M. 2000, ApJ, 528, 552

Küker, M., Rüdifer, G., \& Schultz, M. 2001, A\&A, 374, 301

Markiel, J. A., \& Thomas, J. H. 1999, ApJ, 523, 827

Parker, E. N. 1955, ApJ, 122, 293

Parker, E. N. 1993, ApJ, 408, 707

Schou, J., et al. 1998, ApJ, 131, 442

Spiegel, E. A., \& Zahn, J. P. 1992, A\&A, 265, 106

Tobias, S. M., Weiss, N. O., \& Kirk, V. 1995, MNRAS, 273, 1150

Zhang, K., \& Liao, X. 2003, Chin. J. Astron. Astrophys., 3, 12

Zhang, K., Liao, X., \& Schubert, G. 2003, ApJ, 36, 1124

Zhang, K., Chan, K. H., Zou, J., Liao, X., \& Schubert, G. 2003, ApJ, 596,663 